\title{
Traditional and Latest Researches on Aspergillus oryzae and Related Koji Molds
}

\author{
Yujiro Higuchi ${ }^{1, *(1)}$ and Katsuhiko Kitamoto ${ }^{2}$ \\ 1 Department of Bioscience and Biotechnology, Faculty of Agriculture, Kyushu University, 744 Motooka, \\ Fukuoka 819-0395, Japan \\ 2 Department of Pharmaceutical and Medical Business Sciences, Nihon Pharmaceutical University, Yushima, \\ Bunkyo-ku, Tokyo 113-0034, Japan; k-kitamoto@nichiyaku.ac.jp \\ * Correspondence: y.higuchi@agr.kyushu-u.ac.jp; Tel.: +81-92-802-4734
}

Citation: Higuchi, Y.; Kitamoto, K. Traditional and Latest Researches on Aspergillus oryzae and Related Koji Molds. J. Fungi 2021, 7, 1075. https://doi.org/10.3390/jof7121075

Received: 9 December 2021 Accepted: 13 December 2021 Published: 14 December 2021

Publisher's Note: MDPI stays neutral with regard to jurisdictional claims in published maps and institutional affiliations.

Copyright: (c) 2021 by the authors. Licensee MDPI, Basel, Switzerland. This article is an open access article distributed under the terms and conditions of the Creative Commons Attribution (CC BY) license (https:// creativecommons.org/licenses/by/ $4.0 /)$.
We would like to thank all the contributors to this Special Issue on Aspergillus oryzae and related Koji molds (https://www.mdpi.com/journal/jof/special_issues/Aspergillus_ Koji, accessed on 9 December 2021). Collectively, in this Special Issue, nine reviews and one article have been published to summarize traditional and latest researches about the yellow Koji molds Aspergillus oryzae and Aspergillus sojae, the black Koji mold Aspergillus luchuensis, and the white Koji mold Aspergillus luchuensis mut. kawachii (Figure 1).

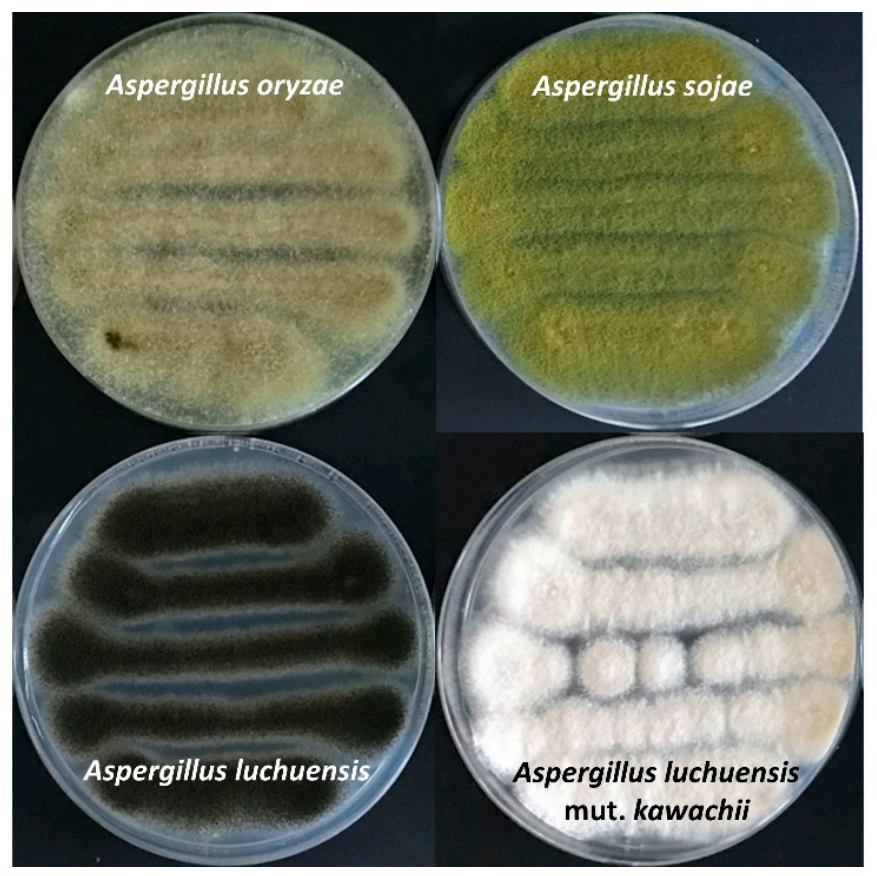

Figure 1. Plate culture images of A. oryzae, A. sojae, A. luchuensis and A. kawachii.

Spores of Koji molds are called Koji starter, and these cultures on cereals and crops are used for fermented foods in industries. Yamashita has outlined the historical backgrounds of Koji starter and Koji, explained these varieties for preparations of fermented foods, and proposed potential uses in the future [1]. One of the fermented beverages produced by using A. oryzae is amazake, the Japanese traditional sweet drink. Kurahashi has provided the information from recent research about ingredients and functionality of amazake [2]. In addition, Kurahashi et al. have reported the latest research data about the improvement of defecation frequency by taking Koji amazake [3]. Shochu and awamori, the Japanese traditional distilled liquors, are made by using A. kawachii and A. luchuensis, 
respectively. Hayashi et al. have described the preparation methods of shochu and awamori with molecular backgrounds and diverse flavor [4]. Miso, a traditional Japanese seasoning paste, is made of soybeans and rice fermented with A. oryzae. Varieties of miso, their preparations with responsible enzymes, and their nutritional components and functions have been covered by Kusumoto et al. [5]. Ito and Matsuyama have detailed the making processes of the Japanese soy sauce, in which Koji molds are essential for producing varieties of crucial enzymes [6]. They have also summarized how glutamate (umami) is generated in soy sauce making. Koji molds are very useful, not only for producing fermented foods and beverages, but also for supplying valuable materials. Kitagaki has listed substances for medical applications that are produced by Koji molds [7]. Due to a great ability in the production of valuable material, including enzyme secretion, the molecular mechanisms of secretory pathways in A. oryzae have been well investigated. Higuchi has covered such recent advances on cell biological aspects of membrane traffic in Koji molds [8]. Not only proteins but also secondary metabolites (SMs) are abundantly produced in Koji molds. Awakawa and Abe have introduced how A. oryzae is a useful heterologous host for producing SMs, such as polyketide-derived meroterpenoids in a group of bioactive natural products [9]. Lastly, Maruyama has covered the genome editing technology CRISPR/Cas9 system in A. oryzae, which has been a powerful molecular tool to develop industrial Koji mold strains [10]. All papers in this Special Issue have provided the traditional and increasing knowledge of $A$. oryzae and related Koji molds with insightful perspectives that will direct future research in each field of study.

Again, we wish to express thanks to all authors and reviewers for their pivotal contributions to this Special Issue, which has been made a highly successful and timely collection of studies.

Funding: This research was funded by JSPS KAKENHI grant number JP19H02874.

Acknowledgments: We would like to thank Norio Takeshita from University of Tsukuba for providing the figure picture.

Conflicts of Interest: The authors declare no conflict of interest.

\section{References}

1. Yamashita, H. Koji starter and Koji world in Japan. J. Fungi 2021, 7, 569. [CrossRef]

2. Kurahashi, A. Ingredients, functionality, and safety of the Japanese traditional sweet drink amazake. J. Fungi 2021, 7, 469. [CrossRef] [PubMed]

3. Kurahashi, A.; Enomoto, T.; Oguro, Y.; Kojima-Nakamura, A.; Kodaira, K.; Watanabe, K.; Ozaki, N.; Goto, H.; Hirayama, M. Intake of Koji amazake improves defecation frequency in healthy adults. J. Fungi 2021, 7, 782. [CrossRef]

4. Hayashi, K.; Kajiwara, Y.; Futagami, T.; Goto, M.; Takashita, H. Making traditional Japanese distilled liquor, shochu and awamori, and the contribution of white and black Koji fungi. J. Fungi 2021, 7, 517. [CrossRef] [PubMed]

5. Kusumoto, K.-I.; Yamagata, Y.; Tazawa, R.; Kitagawa, M.; Kato, T.; Isobe, K.; Kashiwagi, Y. Japanese traditional Miso and Koji making. J. Fungi 2021, 7, 579. [CrossRef] [PubMed]

6. Ito, K.; Matsuyama, A. Koji molds for Japanese soy sauce brewing: Characteristics and key enzymes. J. Fungi 2021, 7, 658. [CrossRef] [PubMed]

7. Kitagaki, H. Medical application of substances derived from non-pathogenic fungi Aspergillus oryzae and A. luchuensis-containing Koji. J. Fungi 2021, 7, 243. [CrossRef] [PubMed]

8. Higuchi, Y. Membrane traffic in Aspergillus oryzae and related filamentous fungi. J. Fungi 2021, 7, 534. [CrossRef] [PubMed]

9. Awakawa, T.; Abe, I. Reconstitution of polyketide-derived meroterpenoid biosynthetic pathway in Aspergillus oryzae. J. Fungi 2021, 7, 486. [CrossRef] [PubMed]

10. Maruyama, J.-i. Genome editing technology and its application potentials in the industrial filamentous fungus Aspergillus oryzae. J. Fungi 2021, 7, 638. [CrossRef] [PubMed] 